\title{
Cyclodextrin/cellulose hydrogel with gallic acid to prevent wound infection
}

\author{
Eva Pinho • Mariana Henriques • Graça Soares
}

Received: 31 March 2014/Accepted: 9 September 2014/Published online: 19 September 2014

(C) Springer Science+Business Media Dordrecht 2014

\begin{abstract}
Cyclodextrin-based hydrogels have been described as suitable for the controlled-release of bioactive molecules to be used as wound dressing. These materials have major advantages, since they gather the hydrogel properties (high degree of swelling and easy manipulation) and the encapsulation ability of cyclodextrins. $\beta$-cyclodextrin $(\beta)$ or hydroxypropyl- $\beta$ cyclodextrin (HP $\beta$ ) was cross-linked (1,4-butanediol diglycidyl ether) with hydroxypropyl methylcellulose under mild conditions. The hydrogels were chemically characterized by swelling degree, FTIR, DSC and contact angle. The gallic acid loading and release was also analysed, as well the antibacterial activity and cytotoxicity of the polymeric networks. The hydrogels obtained were firm and transparent, with good swelling ability. The gel-HP $\beta$ had a surface more hydrophilic when compared with the gel- $\beta$. Nevertheless, both hydrogels were capable to incorporate gallic acid and sustain the release for $48 \mathrm{~h}$. The antibacterial activity of gallic acid was maintained after its adsorption within
\end{abstract}

E. Pinho $(\bowtie) \cdot$ M. Henriques

CEB, Centre for Biological Engineering, LIBRO -

Laboratório de Investigação em Biofilmes Rosário

Oliveira, University of Minho, Campus Gualtar,

4710-057 Braga, Portugal

e-mail: evapinho@deb.uminho.pt

E. Pinho · G. Soares

Centre for Textile Science and Technology (2C2T),

University of Minho, Campus Azurém,

4800-058 Guimarães, Portugal the polymeric matrix, as well as, gallic acid effect on fibroblast proliferation. Therefore, gel- $\beta$ and gel-HP $\beta$ conjugated with gallic acid were shown to be a viable option for antibacterial wound dressing.

Keywords Antibacterial - 1,4-Butanediol diglycidyl ether · Cyclodextrin · Gallic acid · Hydrogel · Wound dressing

\section{Introduction}

Wounds provide suitable environment for the deposition and proliferation of pathogenic microorganisms, causing skin and soft tissue infections, and triggering the patient immunological response (Grice and Segre 2011). The infection severity may range from selflimited superficial to life-threatening diseases. Moreover, skin and soft tissue infections increase the production of wound exudate and tissue deterioration, thus higher wound dressing replacement is required, causing pain to the patient and amplifying the probability of removing the newly formed skin (Chen et al. 2009). Therefore, the research on wound dressings field has been focusing on new wound dressings, capable of mechanical protection and maintaining suitable environmental conditions for proper healing and, additionally, able to perform sustained delivery of antimicrobial agents to prevent wound infections (Pinho et al. 2013).

Hydrogels have been, successfully, applied as wound dressings and drug delivery devices. They are 
polymeric networks with hydrophilic character, capable of absorbing large amounts of water and with suitable physicochemical properties for contact with human tissue without causing injury (Pinho et al. 2013). However, hydrogels have some drawbacks as drug delivery systems. Their capacity to load hydrophobic drugs is quiet reduced, as well as the control over the drug release mechanisms (the diffusion is normally rapid and non-linear) (Thatiparti et al. 2010). So, cyclodextrins (CD)-based hydrogels have been synthesized to improve the drug delivery properties of hydrogels. These materials benefit from the appropriate swelling ability of hydrogels and from the encapsulation capacity of CDs (Rodriguez-Tenreiro et al. 2007). CDs are truncated oligosaccharides with the ability to form inclusion complexes (IC) with a wide range of molecules, due to their hydrophilic surface and hydrophobic cavity. When used as monomer for hydrogel production, CDs can act, simultaneously, as carriers and as enhancers for the hydrogel stability (Pinho et al. 2013).

Nevertheless, the methods used for the preparation of CD-based hydrogels, usually, involve high temperatures leading to toxic bi-products, from undesirable side reactions, reducing their applicability as biomedical devices (Lorenzo et al. 2008). Rodriguez-Tenreiro et al. (2007) developed a method for CD-based hydrogel synthesis with, only 1 step, using condensation with ethylene glycol diglycidyl ether (EGDE) to obtain CD networks, under mild environmental conditions and without previous modification on the $\mathrm{CD}$ structure. CD-based hydrogels synthesized by this method showed good swelling and mechanical properties, and enhanced ability to load and release bioactive molecules (Rodriguez-Tenreiro et al. 2006, 2007; Lorenzo et al. 2008; Blanco-Fernandez et al. 2011; Garcia-Fernandez et al. 2013).

Polyphenolics have been proposed as viable alternatives to broad-spectrum antibiotics, normally, used for the treatment of infected wounds (Wang et al. 2007; Pinho et al. 2014b). Previous work (Pinho et al. 2014a), showed that gallic acid (a simple phenolic acid) has good applicability as antibacterial agent against bacteria, commonly isolated from infected wounds. Although, its applicability on the pharmaceutical field has been constrained by gallic acid susceptibility to environmental factors and low solubility (Guimaraes et al. 2010; Fang and Bhandari 2010; da Rosa et al. 2013).
Therefore, the present work aimed to develop a material capable of forming inclusion complexes with gallic acid and keeping the molecule's antibacterial properties, thus it could be used to prevent wound infections and enhance the healing process.

\section{Materials and methods}

\section{Materials}

Gallic acid (3,4,5-trihydroxybenzoic acid) was provided by Merck, $\beta$-cyclodextrin $(\beta \mathrm{CD}, 1,135 \mathrm{~g} / \mathrm{mol})$ and 2-hydroxypropyl- $\beta$-cyclodextrin $(\mathrm{HP} \beta C D$, $1,309 \mathrm{~g} / \mathrm{mol}$ ) were acquired from AppliChem. Hydroxypropyl methylcellulose (HPMC) Methocel $^{\circledR}$ K4 M (Mw 84,200, normal viscosity 400 mPas) was purchased from VWR Prolab; 1,4-butanediol diglycidyl ether (BDGE, 50-60 \% in water) was obtained from Acros Organics. Purified water, obtained by reverse osmosis $\left(\right.$ MiliQ $^{\circledR}$, Mikipore, Madrid, Spain) with a resistivity above $18.2 \mathrm{M} \Omega / \mathrm{cm}$, was used.

Hydrogel synthesis

The hydrogels were prepared based on the method described by Garcia-Fernandez et al. (2013). Solutions of each $\mathrm{CD}, 2.5 \mathrm{~g}$ in $10 \mathrm{~mL}$ of $\mathrm{NaOH}(0.2 \mathrm{M})$, were prepared and maintained for $5 \mathrm{~min}$ at $25^{\circ} \mathrm{C}$ and $200 \mathrm{rpm}$ (mechanical agitation). The HPMC (0.025 g) was added to each solution and the solubilisation was improved by mechanical agitation $(200 \mathrm{rpm})$ during $5 \mathrm{~min}$ at $25^{\circ} \mathrm{C}$. The solutions $(5 \mathrm{~mL}$ of each) were transferred to petri dishes and $2 \mathrm{~mL}$ of BDGE was added, to each one. The petri dishes were sealed with parafilm and maintained for $2 \mathrm{~min}$ at $25^{\circ} \mathrm{C}$ and under mechanical agitation $(200 \mathrm{rpm})$. To complete the cross-linking process, the plates were kept at $50{ }^{\circ} \mathrm{C}$ for $12 \mathrm{~h}$. At this temperature, the $\mathrm{CD}$ and HPMC stability was assured. After cooling, hydrogels were immersed in ultrapure water for $12 \mathrm{~h}$ and $25^{\circ} \mathrm{C}$, to allow the swelling. Then, they were transferred to an $\mathrm{HCl}$ solution $(10 \mathrm{mM})$ for additional $12 \mathrm{~h}$, and, finally, immersed in water for 7 days. The dry process was performed as followed: the hydrogels were kept at $25^{\circ} \mathrm{C}$ for $24 \mathrm{~h}$ and, after, were transferred to a desiccator until weight stabilization. 
Swelling determination

The amount of water absorbed by the hydrogels was calculated based on the difference between the weight of fully swollen hydrogel in water (W) and the weight after the dry process $\left(\mathrm{W}_{0}\right)$. For that, dry hydrogels $(30 \pm 3 \mathrm{mg})$ were weighted and immersed in $5 \mathrm{~mL}$ of ultrapure water. Hydrogels weight was recorded in regular time periods, until the weight stabilizes (fully swollen hydrogel). For this measurement a Saitouris BL $1,205(\mathrm{~d}=0.1 \mathrm{mg})$ scale was used and the water from the hydrogel surface was removed before weighing. The swelling profile resulted from the data obtained, by plotting the amount of water absorbed at time $\mathrm{t}\left(\mathrm{W}_{\mathrm{t}}\right) /$ amount of water at equilibrium $(\mathrm{W} \infty)$ ) versus time (min).

The degree of swelling (Q) was calculated based on Eq. 1.

$Q=\frac{W-W_{o}}{W_{o}} \times 100$

Gallic acid loading and release

Gallic acid solution $\left(2.3 \times 10^{-2} \mathrm{M}\right.$, in $2 \%$ methanol $)$ was dissolved in $\mathrm{H}_{3} \mathrm{PO}_{4} / \mathrm{NaOH}$ buffer $(\mathrm{pH} 3 \pm 0.5)$, and kept for $30 \mathrm{~min}$ in an ultrasonic bath, to allow its complete solubilisation. Dry hydrogel samples (30 $\pm 3 \mathrm{mg}$ ) were immersed in $5 \mathrm{~mL}$ of gallic acid solution at $25{ }^{\circ} \mathrm{C}$ and $60 \mathrm{rpm}$. The amount of phenolic acid in the solution was assessed by UV-Vis spectrophotometry, until the absorbance values stabilize. The gallic acid loading was calculated based on the variation of gallic acid in the initial solution and at the equilibrium.

The gallic acid release, from hydrogels, was performed using load dry samples. The samples $(30 \pm 3 \mathrm{mg})$ were immersed in $5 \mathrm{~mL}$ of synthetic sweat solution (SSS, $0.5 \mathrm{~g}$ L-histidine monohydrochloride monohydrate, $5 \mathrm{~g}$ of sodium chloride, $2.2 \mathrm{~g}$ of sodium dihydrogen orthophosphate dihydrate, $\mathrm{pH}$ $5 \pm 0.5$ ). The samples were maintained at $25^{\circ} \mathrm{C}$ and the absorbance of the released medium was monitored until stabilization.

The absorbance was measured at the gallic acid $\lambda_{\max }(259 \mathrm{~nm}$ for $\mathrm{pH} 3, \varepsilon 2.30 \mathrm{M} / \mathrm{cm}$ or $261 \mathrm{~nm}$ for $\mathrm{pH}$ $5, \varepsilon 4.27 \mathrm{M} / \mathrm{cm})$. The gallic acid concentration was calculated based on the calibration curve, previously determined. All the absorption measurements were recorded on a Jasco V560 spectrometer, using a $1 \mathrm{~cm}$ quartz cuvette.
Hydrogels physicochemical characterization

Fourier transform infrared spectroscopy (FTIR)

Dry samples $(0.5 \pm 0.05 \mathrm{~g})$ of each hydrogel, $\beta \mathrm{CD}$ co-HPMC (gel- $\beta$ ) and HP $\beta C D-c o-H P M C ~(g e l-H P \beta)$ with or without gallic acid, were used for FTIR-ATR analysis. The spectra were recorded between 400 and $4,000 \mathrm{~cm}^{-1}$, in Avatar 360 FTIR spectrometer. The spectra of individual compounds (gallic acid, $\beta C D$, HP $\beta C D$ and HPMC) were, also, recorded, using the potassium bromide pellet technique.

Thermal analysis by differential scanning calorimetry (DSC)

DSC measurements were carried in liquid nitrogen atmosphere using DSC-822e instrument (Mettler Toledo). The calibration was made with indium as standard. Dry samples were weighed $(2.5 \pm 0.2 \mathrm{mg})$ and sealed in aluminium pans. Then, they were heated from $25^{\circ}$ to $350{ }^{\circ} \mathrm{C} / \mathrm{min}$, at a scanning rate of $10^{\circ} \mathrm{C} \cdot \mathrm{min}^{-1}$. Data were treated using LAB mettler star SW 8.1 software (Mettler-Toledo International Inc, Swiss).

\section{Contact angle}

Water contact angles of hydrogels were determined using a contact angle measurement apparatus (OCA15 Plus; Dataphysics, Germany). A water drop ( $3 \mu \mathrm{L})$ was placed over the clean hydrogel surfaces with an autopipette. All measurements were performed at room temperature.

All the characterization methods were made in triplicate for each hydrogel (gel- $\beta$, gel- $\beta /$ gallic acid, gel-HP $\beta$, gel-HP $\beta /$ gallic acid).

In vitro analysis of hydrogels' biological properties

\section{Antibacterial activity}

The hydrogels' antibacterial activity was tested against 3 bacteria: Staphylococcus epidermidis (ATCC 12228), Staphylococcus aureus (ATCC 6538) and Klebsiella pneumoniae (ATCC 11296). The bacteria were grown in tryptic soy agar (TSA, Merck, Germany) for $24 \mathrm{~h}$ at $37{ }^{\circ} \mathrm{C}$. The cells were 
inoculated in tryptic soy broth (TSB, Merck, Germany) and incubated for $18 \mathrm{~h}$ at $37{ }^{\circ} \mathrm{C}$ under agitation (120 rpm). Subsequently, bacterial concentration of each strain was adjusted to $1 \times 10^{6}$ cells $/ \mathrm{mL}$, via absorbance readings and the corresponding calibration curve.

The hydrogels' antimicrobial activity was measured both qualitative and quantitatively. For the first analysis, the disc diffusion method described by the National Committee for Clinical Laboratory Standards (NCCLS), M2-A8 document (2005), was used with some modifications. The TSA was the nutritive media used and $200 \mu \mathrm{L}$ of each inoculum $\left(1 \times 10^{6}\right.$ cells $/ \mathrm{mL}$ ) was spread on the media plates. The hydrogel samples $(30 \pm 3 \mathrm{mg}$ and with approximately $1 \pm 0.2 \mathrm{~mm}$ of diameter) were placed over the petri dish. The plates were then incubated at $37{ }^{\circ} \mathrm{C}$ for $18 \mathrm{~h}$. The size of the inhibition halo was measured.

For the quantitative assays, samples $(30 \pm 3 \mathrm{mg})$ were immersed in $5 \mathrm{~mL}$ of $5 \times 10^{5}$ cells $/ \mathrm{mL}$ of each bacterium. Bacteria and medium controls were, also, included. The plates were incubated for $18 \mathrm{~h}$ at $37^{\circ} \mathrm{C}$. The number of viable cells, was assessed by determination of the number of colony forming units (CFUs), by plating $10 \mu \mathrm{L}$ of cell suspension from each well onto TSA, and incubated for $24 \mathrm{~h}$ at $37{ }^{\circ} \mathrm{C}$.

Both methods were made in triplicate for each bacterium in, at least, 3 independent assays.

\section{Hydrogels effect on fibroblast proliferation}

Hydrogels' cytotoxicity in vitro assay was carried out based on the method described on ISO 10993-5:2009 Biological evaluation of medical devices, part 5: Tests for in vitro cytotoxicity, by indirect contact.

The liquid extracts of the hydrogel were prepared as follows: hydrogels $(30 \pm 3 \mathrm{mg})$ were immersed in $5 \mathrm{~mL}$ of Dulbecco's modified Eagle's medium (DMEM), and then they were kept $18 \mathrm{~h}$ at $25^{\circ} \mathrm{C}$ in the dark.

Fibroblast 3T3 (CCL 163-from American Type Culture Collection) were used for this study. Cells were cultured in DMEM supplemented with $10 \%$ of foetal bovine serum and $1 \%$ penicillin/streptomycin at $37{ }^{\circ} \mathrm{C}, 5 \% \mathrm{CO}_{2}$. After achieving confluence, cells were passed at the density of $1 \times 10^{5}$ cells $/ \mathrm{mL}$, using trypsin. Cells were seeded at the density of $5 \times 10^{5}$ cells/mL (48 well plate) in $300 \mu \mathrm{L}$ of DMEM complete medium.
The cytotoxicity was tested through the MTS ([3(4,5-carboxymethoxyphenyl)-2-(4-sulfophenyl)- $2 \mathrm{H}$ tetrazolium]) assay. For that, the medium was replaced with $300 \mu \mathrm{L}$ of the liquid extract of hydrogels and the cells were incubated at $37{ }^{\circ} \mathrm{C}$ and $5 \% \mathrm{CO}_{2}$. After $24 \mathrm{~h}$, the medium was removed and a mixture of $6 \mu \mathrm{L}$ of MTS (Promega) and $294 \mu \mathrm{L}$ of DMEM without phenol was added to each well. After $1 \mathrm{~h}$, the absorbance value was measured at $490 \mathrm{~nm}$ and the results were expressed as percentage of viable cells $(\%)$, using the number of cells grown on wells without hydrogel $($ control + ) as $100 \%$.

All the mathematical analyses were made using the Origin Pro software.

\section{Results and discussion}

The gallic acid encapsulation by $\beta C D$ and HP $\beta C D$ has been studied previously by our group (Pinho 2014). The ICs obtained showed good stability parameters and the gallic acid ability to destroy bacteria, usually isolated from skin and soft tissue infections, was preserved. Thus, these CDs were used for the CDbased hydrogel synthesis using HPMC (a cellulose derivative), and were cross-linked with BDGE. The HPMC is a linear polymer constituted by glycopyranose units, similar to those found on CDs. HPMC has been used in hydrogels due to its high swellability and biocompatibility (Siepmann and Peppas 2001; Zugasti et al. 2009). Hydrogels were made under alkaline $\mathrm{pH}$ and mild temperature as described by Lorenzo et al. (2008).

The attempts to prepare hydrogels with $\beta C D$ or HP $\beta C D$ cross-linked with BDGE (without HPMC), following the same protocol failed, since they did not form gels. Other authors (Rodriguez-Tenreiro et al. 2006, 2007; Blanco-Fernandez et al. 2011) reported the formation of HP $\beta C D$ hydrogels using EGDE as cross-linking agent within similar conditions. In the present work, BDGE was used as cross-linking agent instead of EDGE. Both molecules have 2 glycidyl groups, capable of reacting, simultaneously, with the CDs' hydroxyl group or with cellulose ether group. They differ in size of the chain between the epoxy groups, BDGE has a longer chain which increases the distance between the $\mathrm{CD}$ and cellulose molecules (Lorenzo et al. 2008). Thus, it was expected to obtain a more flexible hydrogel without losing the non-toxic 


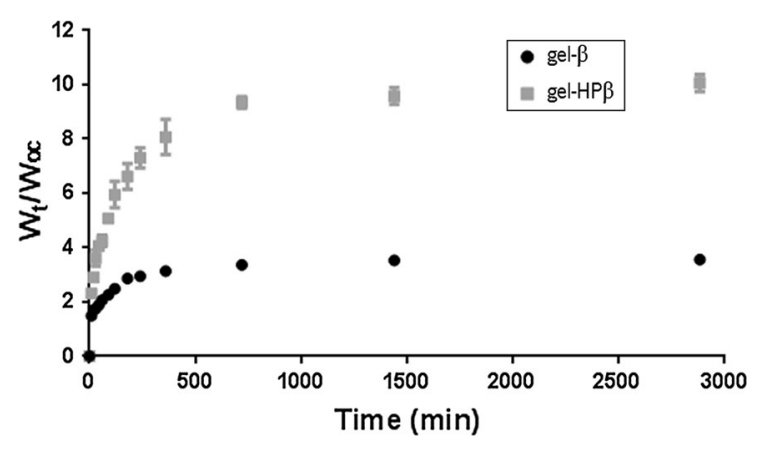

Fig. 1 Swelling profiles in water of gel- $\beta$ (circle) and gel-HP $\beta$ (square) dry hydrogels prepared with $25 \%$ cyclodextrin/ $/ .25 \%$ HPMC

behaviour of the cross-linking agent (Nicoletti et al. 2013). However, this characteristic may, also, cause the lack of suitable mechanical properties observed in the hydrogels without HPMC.

The CD-based hydrogels were cross-linked with BDGE in the presence of HPMC and 1:1.25:0.1 ratio between compounds was maintained, since it was described as the best condition by Rodriguez-Tenreiro et al. (2006). The hydrogels obtained with HPMC (gel$\beta$ and gel-HP $\beta$ ) were transparent and easy to handle, with smooth and continuous surface. They, also, had enough elasticity, suitable for direct contact with injured skin (Lorenzo et al. 2008). Additionally, the gel- $\beta$ was more transparent and with lower elasticity than the gel-HP $\beta$. After immersion in water, both hydrogels kept their shape and behaved as superabsorbent systems. All these features point out to the formation of a homogeneously cross-linked hydrogels (Rodriguez-Tenreiro et al. 2006).

\section{Swelling ability}

Swelling represents the water holding ability and permeability of hydrogels. The hydrogels swelling starts with the diffusion of water molecules into the network, hydration of polar hydrophilic groups and polymer expansion until the free water molecules and the molecules within the network reach equilibrium (Zhang et al. 2005; Gulrez et al. 2011).

The swelling profiles (Fig. 1) show that both hydrogels had a fast swelling during the first $400 \mathrm{~min}$ and the equilibrium was reached after 700 min. Figure 1 demonstrated that gel-HP $\beta$ was

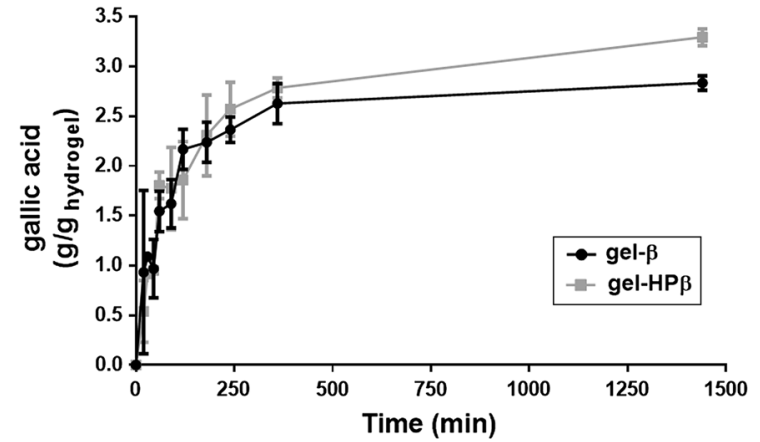

Fig. 2 Gallic acid loading (a) in gel- $\beta$ (black) and gel-HP $\beta$ (grey). The loading was performed during $24 \mathrm{~h}, 25^{\circ} \mathrm{C}$ with gallic acid dissolved on the buffer $\mathrm{H}_{3} \mathrm{PO}_{4} / \mathrm{NaOH}(\mathrm{pH} 3 \pm 0.5)$

capable of taking more quantity of water molecules than the gel- $\beta$. In addition, the $Q$ values show that gelHP $\beta$ absorbed 9 times more its weight in water, and the gel- $\beta$ only 2.5 times (data not shown). Similar swelling profiles were reported for CD- based hydrogels HPMC (Rodriguez-Tenreiro et al. 2006, 2007; Zhang et al. 2013).

Based on the swelling profiles, both hydrogel networks are suitable for water and small size molecule diffusion. However, the gel- $\beta$ had less affinity for water, probably related with higher degree of cross-linking or with overlapped HPMC chains within the network (Lorenzo et al. 2008). On the contrary, the hydroxypropyl groups of HP $\beta C D$ increase the network pore size, improving the network ability to absorb water molecules.

\section{Gallic acid loading}

Hydrogels ability to retain molecules depends on (1) the network degree of cross-linking, (2) the water affinity of the polymeric molecules, and (3) interactions between the guest and the network components. All these factors regulate the molecule capability to diffuse within the hydrogel (Challa et al. 2005).

Figure 2 displays the quantity of gallic acid incorporated within hydrogels. The loading was performed with gallic acid dissolved in $\mathrm{H}_{3} \mathrm{PO}_{4} / \mathrm{NaOH}$ buffer at $\mathrm{pH} 3$, since it was previously defined as the best condition for the IC formation between this phenolic acid and $\beta C D$ or HP $\beta C D$ (Pinho 2014).

The hydrogel rate of antibacterial agent incorporation was similar to the swelling profile. The 

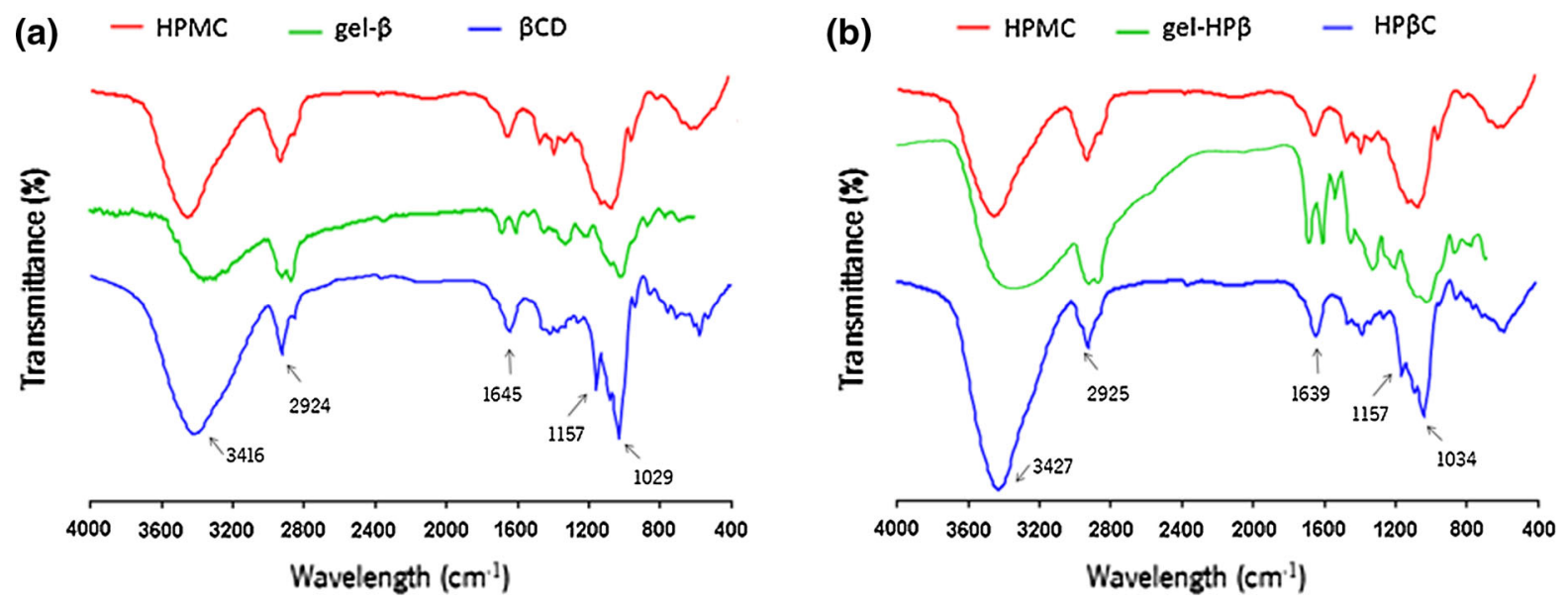

Fig. 3 FTIR spectra CD (blue) and HPMC (red) powders, gel (green). a with $\beta C D$ and b with HP $\beta C D$. (Color figure online)

equilibrium between the free gallic acid and within the hydrogel was achieved after $6 \mathrm{~h}$. Nevertheless, the difference between the 2 hydrogels obtained for the swelling was not so obvious in the case of gallic acid loading. Thus, both hydrogels showed similar ability to incorporate gallic acid (gel- $\beta 2.76 \mathrm{~g} / \mathrm{g}_{\text {hydrogel }}$ and gel-HP $33 \mathrm{~g} / \mathrm{g}_{\text {hydrogel }}$ ).

In the present work, both hydrogels had similar loading capacity suggesting that the gallic acid encapsulation by cyclodextrins was crucial for the gallic acid loading by the networks. $\beta C D$ and $\mathrm{HP} \beta \mathrm{CD}$ were described as suitable for the encapsulation of poorly soluble gallic acid with 1:1 stoichiometry and good stability parameters [stability constant (K) 40 and $90 \mathrm{M}^{-1}$, respectively, (Pinho 2014)]. The gelHP $\beta$ loading capacity was superior, in accordance with the higher ability of HP $\beta C D$ to encapsulate gallic acid. Therefore, gel-HP $\beta$ kept the ability to form IC with higher stability than the native $\mathrm{CD}$, since the gallic acid loading was higher for this hydrogel. Moreover, the gallic acid could also be trapped on the aqueous phase of the gel-HP $\beta$, since this polymeric network showed higher swelling ability.

Hydrogel characterization

\section{FTIR analysis}

The hydrogels cross-linking was characterized based on the FTIR spectra of dried hydrogels and powders CDs and HPMC (Fig. 3). As expected, the spectra from both CD were similar, as result of their truncated shape with one edge lined with primary hydroxyl groups $(\mathrm{OH})$ and the other edged with secondary groups. On their spectra, the following peaks were identified $(\beta C D / H P \beta C D): 3,416 / 3,447 \mathrm{~cm}^{-1}(\mathrm{O}-\mathrm{H}$ stretching), $2,924 \mathrm{~cm}^{-1}$ (stretching vibrational asymmetric of C-H), 1,645/1,640 $\mathrm{cm}^{-1}$ (hydrogen interactions), $1,157 \mathrm{~cm}^{-1}$ (C-O stretching) and 1,029/ $1,034 \mathrm{~cm}^{-1}$ (C-O-C) (Rodriguez-Tenreiro et al. 2006; Schwingel et al. 2008; Zhang et al. 2013). The HPMC spectra showed a profile similar to the CD and the stronger intensity peaks identified were: $3,447 \mathrm{~cm}^{-1}$ (O-H stretching), 2,924 $\mathrm{cm}^{-1}$ (stretching vibrational asymmetric of $\mathrm{C}-\mathrm{H}$ ), $1,640 \mathrm{~cm}^{-1}$ (hydrogen interactions), 1,115 and $1,063 \mathrm{~cm}^{-1}$ (ether bond) (Sun et al. 2003; Miranda et al. 2010).

The hydrogels spectra had similar profile regardless of the CD used for the synthesis of the polymeric network. However, they display some differences in the peaks intensity and shape when compared with the CDs and HPMC spectra. For instances, the peak attributed to the ether bonds (between 1,200 and $1,000 \mathrm{~cm}^{-1}$ ) became weak and with a broad shape, as consequence of the decrease of those bonds induced by the cross-linking reaction. The intensity of the peaks near 3,400 and $1,649 \mathrm{~cm}^{-1}$ decreased due to the reduction of $\mathrm{OH}$ groups and hydrogen bonds, after the cross-linking (Rodriguez-Tenreiro et al. 2006; Zhang et al. 2013). Thus, the majority of the CDs' $\mathrm{OH}$ were efficiently linked to the HPMC by the BDGE. Moreover, no peak was detected near $1,250 \mathrm{~cm}^{-1}$ meaning that no free BDGE was within hydrogels. 

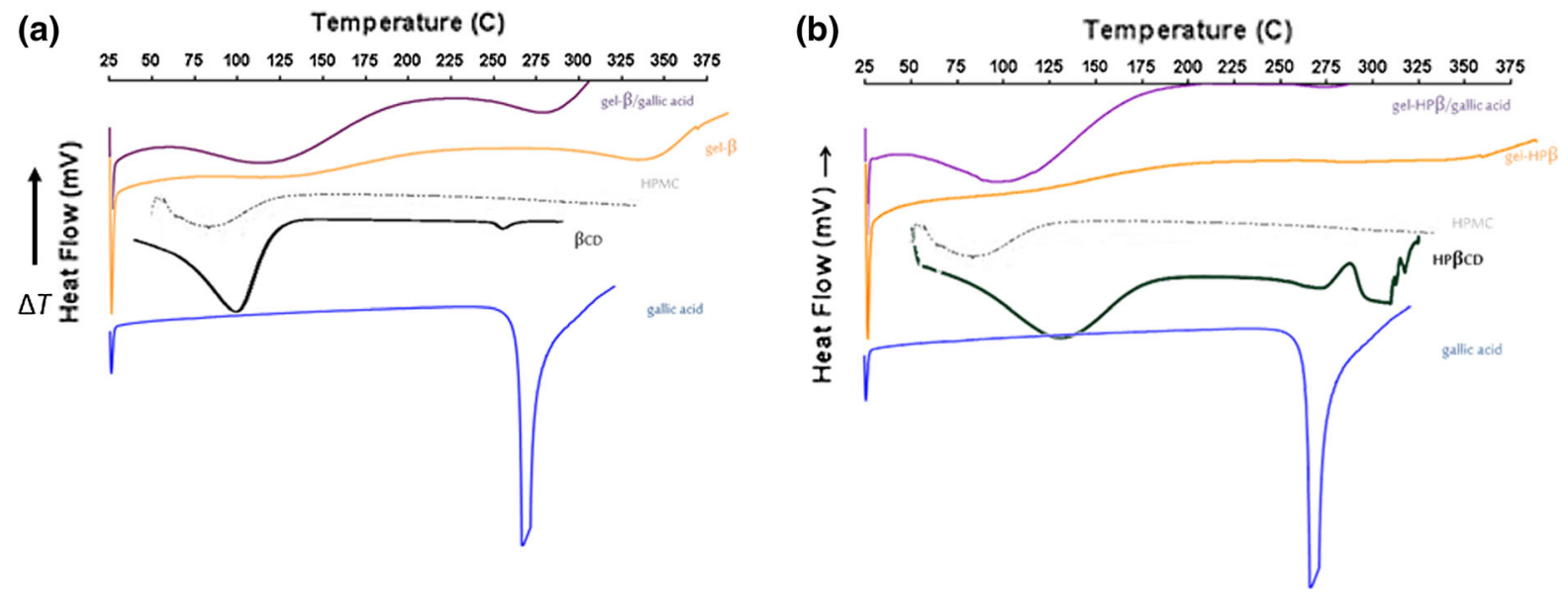

Fig. 4 DSC thermograms of gallic acid (blue), CD (black), HPMC (grey), gel (orange) and gel loaded (purple). a with $\beta C D$ and b with $\mathrm{HP} \beta \mathrm{CD}$. (Color figure online)

\section{Thermal analysis}

Hydrogels synthesis induced alterations on thermal properties of each molecule ( $\beta C D, H P \beta C D$ and HPMC), as result of the interactions established to form the polymeric network. DSC thermograms display the intermolecular interactions between hydrogels components within the network (Ciolacu et al. 2012). Figure 4 shows that gallic acid has a sharp endothermic peak $\left(264{ }^{\circ} \mathrm{C}\right)$, as well as the $2 \mathrm{CDs}(2$ peaks near 100 and $275^{\circ} \mathrm{C}$ ). The HPMC exhibits a smaller and broad peak near $75^{\circ} \mathrm{C}$. The cross-linking between HPMC and CDs induces obvious alterations on the DSC thermograms profiles. The profiles of both hydrogels (gel- $\beta C D$ and gel-HP $\beta$ ) have a broad peak, confirming the crosslinking between the 2 compounds, and suggesting the presence of an amorphous structure, characteristic of these materials. Moreover, the thermogram of the hydrogels that were loaded with gallic acid, also, lacks the phenolic acid peak. Thus, the gallic acid may be trapped in an amorphous or solid solution state within the polymeric network (Dandekar et al. 2010).

\section{Surface hydrophilicity measurement}

The hydrogel wettability or hydrophilicity plays a major role on biocompatibility, since the polymer surface states the interaction between the living system and the medical device (Wang et al. 2004). This property can be set by the measurement of the contact angle formed between water and the hydrogel.
Table 1 Contact angle obtained with water when in contact with the hydrogels surface

\begin{tabular}{llr}
\hline Contact angle $\left({ }^{\circ}\right)$ & Gel- $\beta$ & \multicolumn{1}{c}{ Gel-HP $\beta$} \\
\hline Without gallic acid & $85.68 \pm 1.33$ & $97.37 \pm 1.63$ \\
With gallic acid & $73.83 \pm 1.27$ & $106.82 \pm 2.86$ \\
\hline
\end{tabular}

The values obtained with gel- $\beta$ and gel-HP $\beta$ with or without gallic acid are summarized in Table 1 .

Regarding gel- $\beta$, the contact angle measured was lower than $90^{\circ}$, thus this network has a slightly hydrophilic surface (Jones et al. 2008). Moreover, gel$\beta$ contact angle decreased after the gallic acid loading. An opposite behaviour was detected for gel-HP $\beta$. This hydrogel displayed a higher contact angle than the hydrogel with the native $\mathrm{CD}$, and the gallic acid loading increased the hydrophobicity of its surface.

The hydrogel major characteristic is the ability to absorb large amounts of water, so it should be expected to obtain low contact angles, due to hydrogel hydrophilic surface. However, the macromolecules within the networks hold high mobility to rearrange their orientation according to the environment. Thus, in the specific case of hydrogels, the contact angle value reflects the degree of freedom of the network molecules, to move as response to the environment change (Yasuda et al. 1981).

According to the swelling and gallic acid loading results (Figs. 1 and 2), it was predictable that the gelHP $\beta$ had lower contact angle, when compared with 


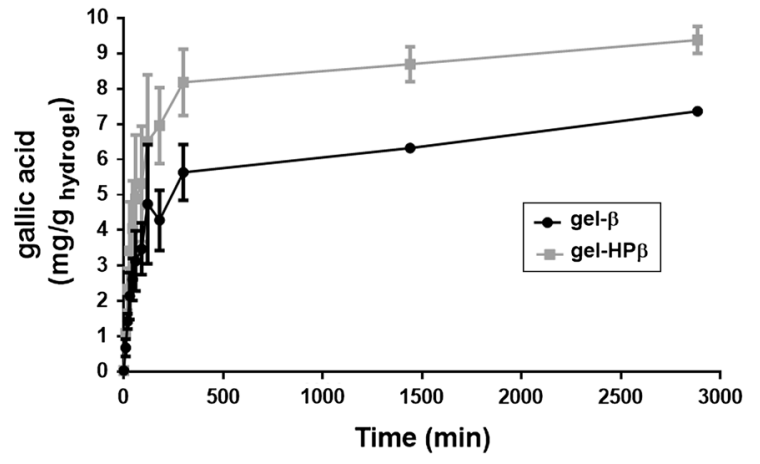

Fig. 5 Gallic acid release from gel- $\beta$ (black) and gel-HP $\beta$ (grey) hydrogels, it was performed during $48 \mathrm{~h}, 25^{\circ} \mathrm{C}$ within synthetic sweat solution $(\mathrm{pH} 5 \pm 0.1)$

gel- $\beta$. The apparent hydrophobicity observed for this hydrogel surface may be caused by the less freedom of the network to rearrange their macromolecules, due to the hydroxypropyl groups (Yasuda et al. 1994). These groups increase the molecules size, which enlarge the mesh size (higher swelling), but decrease the molecules mobility within the network (low hydrophilic surface). Moreover, the HP $\beta C D$ has the ability to establish an IC with gallic acid with higher stability parameters than the $\beta C D$ (Pinho 2014). Thus, the gallic acid interaction with this cyclodextrin will be stronger, contributing to the minor interaction of the phenolic acid with water, and, consequently, the degree on hydrophilicity of gel-HP $\beta$ network.

\section{Gallic acid release from hydrogels}

Figure 5 displays the gallic acid release profiles in SSS; this solution was chosen to simulate the behaviour of the hydrogels in contact with the skin. Both hydrogels were capable of controlling the gallic acid release for 2 days, time at which the equilibrium between the gallic acid within the network and in solution was achieved. Additionally, the release profiles were also analogous for the 2 networks, there was a burst release during the first $6 \mathrm{~h}$, as result of gallic acid migration from the hydrogels surface to the solution. The slower release, after $6 \mathrm{~h}$, can be ascribed to the phenolic compound dissociation from the CDs, followed by its diffusion through the network. Moreover, the amount of gallic acid released was higher for the gel-HP $\beta$, as result of the greater loading and swelling capacity of this network, as referred above.
Biological activity of hydrogels

\section{Antibacterial activity}

Gallic acid antibacterial activity has been attributed to its interaction with bacteria cells' surfaces (enhanced by gallic acid affinity to the lipophilic membrane layer), which induced alterations on the cell electrochemical potential, and reduced the membrane integrity. The gallic acid uptake creates a hyperacidification of cytoplasm, via proton donation, interfering with crucial metabolic pathways (Borges et al. 2013). Previous research, made by the group, concluded that gallic acid antibacterial activity was conserved after encapsulation by $\beta C D$ and HPßCD (Pinho 2014).

The main goal of the present work was to develop a hydrogel capable of preventing the deposition and growth of bacteria on the wound site. Hence, the polymeric networks antibacterial activity was evaluated by qualitative and quantitative methods. Figure 6 shows the growth inhibition halos obtained for the gallic acid loaded hydrogels. The gallic acid maintained its ability to reduce the bacteria growth.

The quantification of the hydrogels' antibacterial activity, against the 3 bacteria (Fig. 7), was consistent with the previous analysis (Fig. 6). The gel- $\beta$ and gelHP $\beta$ were able to destroy all the bacterial cells, with the exception of the gel-HP $\beta$ when in contact with $S$. aureus. Although, in this case the growth observed was minimal (only $1 \mathrm{log}$ ).

Therefore, it was verified that the gallic acid antibacterial activity was preserved after incorporation within the hydrogels. Considering that the hydrogels samples had $30 \mathrm{mg}$, and the gallic acid release (gel- $\beta 7.4 \mathrm{mg} / \mathrm{g}_{\text {hydrogel }}$ and gel-HP $\beta 9.4 \mathrm{mg} /$

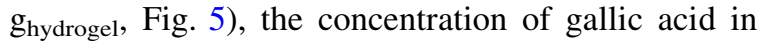
contact with bacteria would be, at least, $0.26 \mathrm{mM}$ for gel- $\beta$ and for gel-HP $\beta 0.33 \mathrm{mM}$. These values are lower than minimal bactericidal concentration reported $(0.47 \mathrm{mM})$ (data not shown). Despite that, both networks were capable of significantly decreasing the growth of all three bacteria. Thus, antibacterial activity of hydrogels was based, not only on the amount of phenolic acid capable to migrate from the hydrogel to the solution, but also on the amount of gallic acid trapped inside the hydrogels. 
Fig. 6 Qualitative analysis of the gallic acid loaded hydrogels antibacterial activity against 3 bacteria (K. pneumoniae, $S$. epidermidis and $S$. aureus, $1 \times 10^{6}$ cells $\mathrm{mL}^{-1}$ )

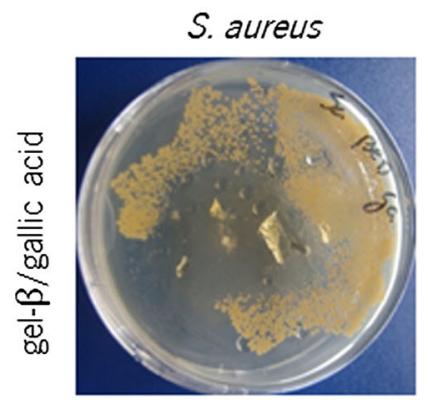

\section{S. epidermidis}
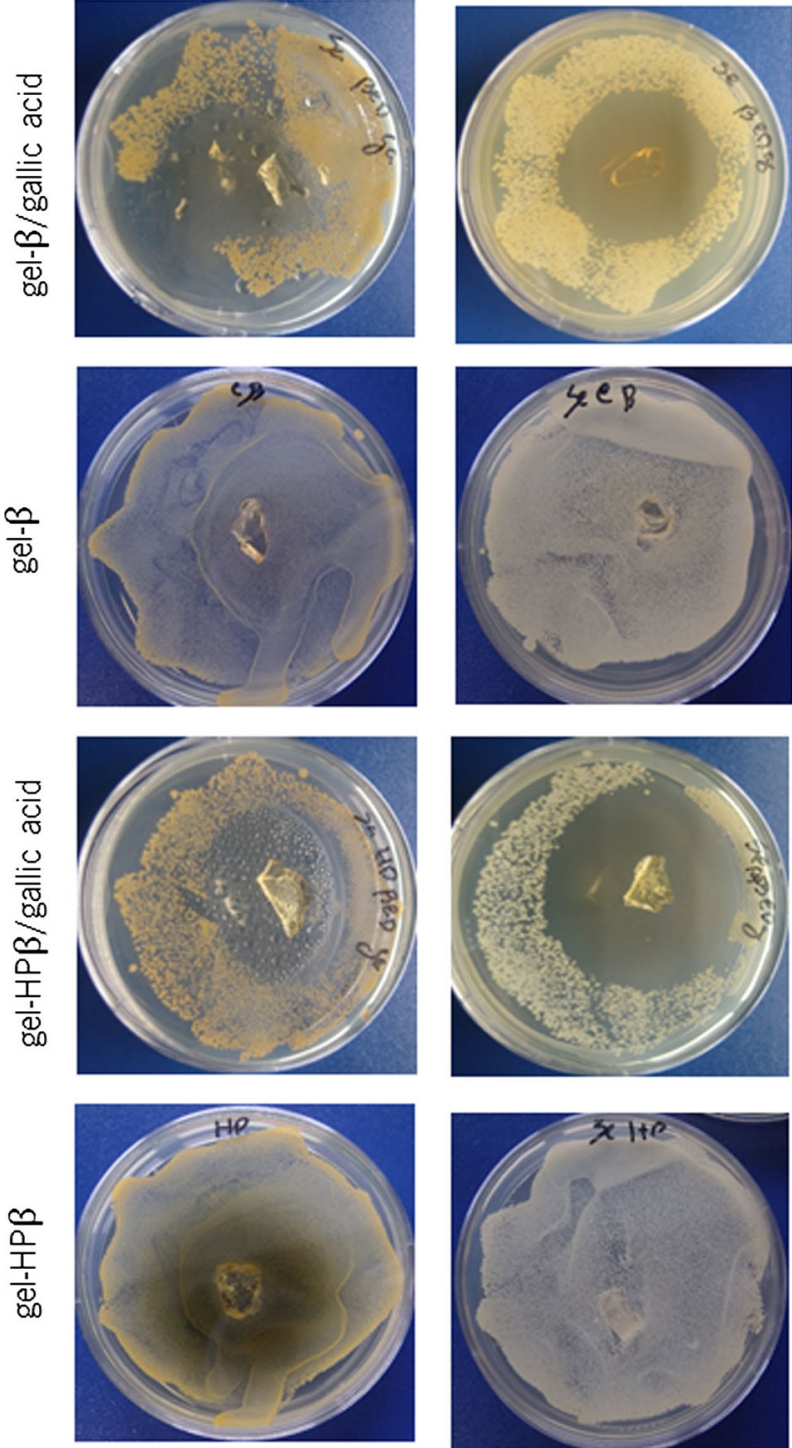
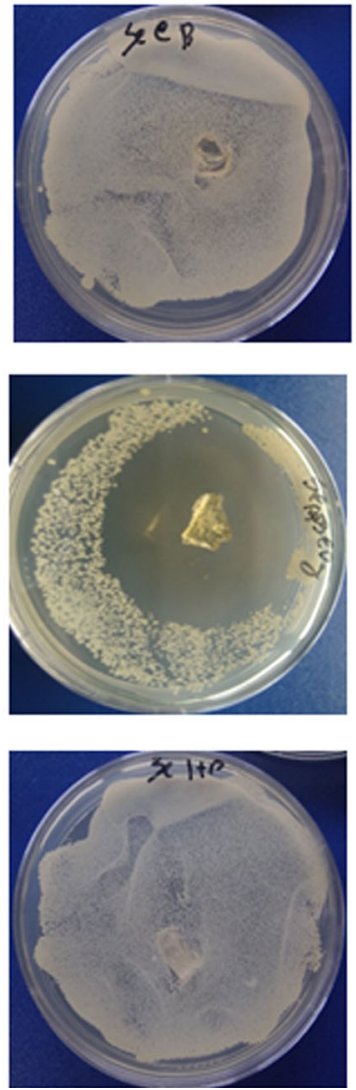

K. pneumoniae
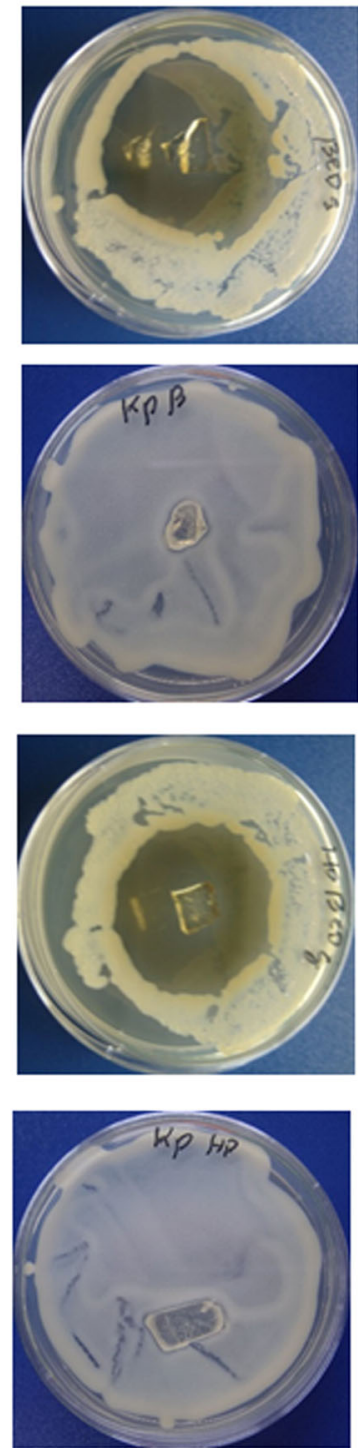

\section{Effect of hydrogels on fibroblast proliferation}

The successful utilization of gel- $\beta$ and gel-HP $\beta$ as wound dressing, also, depends on their biocompatibility. Biocompatibility reflects the interaction between the artificial material and tissues, and it can be evaluated by in vitro cytotoxicity (Wang et al. 2004). In the present study, MTS test was used to evaluate the cytotoxicity effect of the new developed hydrogels. The MTS assay allowed the measurement of cellular viability, due to cell capacity to uptake
MTS and its subsequent reduction by the mitochondria, leading to alterations in MTS colour.

The percentage of viable cells (Fig. 8) measured after contact with the extracts from gel- $\beta$ and gel-HP $\beta$ were similar to the percentage of the normal conditions of growth (control + ). Thus, both hydrogels do not release any kind of substance that could be potentially hazardous for fibroblast.

The gallic acid induces a decrease in the viable cells (Fig. 8). It was previously shown that gallic acid can enhance fibroblasts proliferation when applied at low 


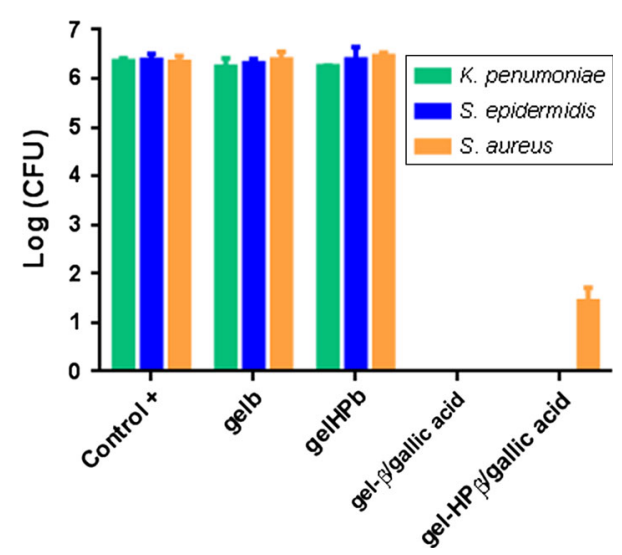

Fig. 7 Quantitative analysis of antibacterial activity of the gallic acid loaded hydrogels by direct contact against 3 bacteria (K. pneumoniae, S. epidermidis and $S$. aureus, $5 \times 10^{5}$ cells $\mathrm{mL}^{-1}$ )

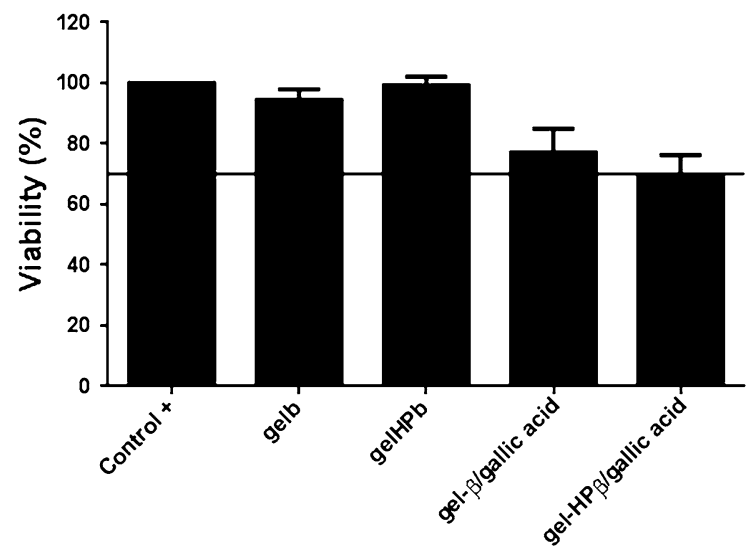

Fig. 8 Viability of fibroblast 3T3 after $24 \mathrm{~h}$ of contact with liquid extracts from hydrogels ( $24 \mathrm{~h}$ within DMEM), measured with MTS assay. The control + allowed the perfect growth of the cells. All data is expressed as mean + standard deviation $(\mathrm{n}=9)$. The line indicates $70 \%$ of cell viability

concentrations, and induces a reduction on viable cells for concentrations above $0.6 \mathrm{mM}$ (Pinho et al. 2014a). Assuming that, at least, 0.26 or $0.33 \mathrm{mM}$ of gallic acid was released to the medium used for contact with fibroblast. The gallic acid effect on fibroblast proliferation was similar to the one described for the free gallic acid.

Thus, the gallic acid biological properties were not affected by its incorporation within both hydrogels. Moreover, the percentage of viable cells, for both hydrogels, was above the limit described as safety for humans (70 \%), based on ISO 10993-5:2006.
Therefore, gel- $\beta$ and gel-HP $\beta$ conjugated with gallic acid may be a viable option for wound dressing without causing any damage to the surrounding tissue.

\section{Conclusion}

The CD-based hydrogels obtained from the crosslinking between $\beta C D$ or HP $\beta C D$ and HPMC were developed to be applied as wound dressings, capable of preventing wound infections. To the best of our knowledge, loading and release of gallic acid (as antibacterial agent) into hydrophilic networks of $\mathrm{CD}$, HPMC and BDGE (as cross-linker), and its release for control wound infections, have not been evaluated until now.

The hydrogels obtained, after successful crosslinking with BDGE under mild conditions, were transparent, easy to handle and soft, thus suitable for the contact with injured skin. Gel- $\beta$ and gel-HP $\beta$ behaved as superabsorbent hydrogels, being the last network capable of higher swelling. The swelling and gallic acid loading profiles were similar. The results obtained (DSC, FTIR and release) suggested that gallic acid may be inside the cyclodextrins cavity and, also, trapped in the polymeric network. The gel-HP $\beta$ has a less hydrophilic surface when compared with the gel- $\beta$, as result of the lower mobility of its network. Regarding the biological properties of both hydrogels, the gallic acid antibacterial activity was preserved after its incorporation within the hydrogels. In addition, all the hydrogels, with or without gallic acid, enabled the fibroblast proliferation.

In conclusion, the gallic acid was successfully loaded into the polymeric network produced, and its release was sustained for $48 \mathrm{~h}$. Moreover, the loaded gel- $\beta$ and gel-HP $\beta$ were capable of destroying bacterial cells preserving the gallic acid antibacterial activity. Based on the results from the present work, the gel-HP $\beta$ appears to be the network with more suitable properties for the incorporation of gallic acid and utilization as antibacterial wound dressing, without causing any damage to the surrounding tissue.

Acknowledgments The authors thank the FCT Strategic Projects PEst-OE/EQB/LA0023/2013, PEst-C/CTM/UI0264/ 2011, the Project "BioHealth-Biotechnology and Bioengineering approaches to improve health quality", Ref. NORTE07-0124-FEDER-000027, co-funded by the Programa Operacional Regional do Norte (ON.2-O Novo Norte), QREN, FEDER, and E. Pinho grant (SFRH/BD/62665/2009). 


\section{References}

Blanco-Fernandez B, Lopez-Viota M, Concheiro A, AlvarezLorenzo C (2011) Synergistic performance of cyclodextrin-agar hydrogels for ciprofloxacin delivery and antimicrobial effect. Carbohydr Polym 85:765-774. doi:10.1016/ j.carbpol.2011.03.042

Borges A, Ferreira C, Saavedra MJ, Simões M (2013) Antibacterial activity and mode of action of ferulic and gallic acids against pathogenic bacteria. Microb Drug Resist 19:256-265. doi:10.1089/mdr.2012.0244

Challa R, Ahuja A, Ali J, Khar RK (2005) Cyclodextrins in drug delivery: an updated review. Aaps Pharmscitech 6:329357

Chen L, Wu J, Yuwen L et al (2009) Inclusion of tetracycline hydrochloride within supramolecular gels and its controlled release to bovine serum albumin. Langmuir 25:8434-8438. doi:10.1021/la8043208

Ciolacu D, Oprea AM, Anghel N et al (2012) New celluloselignin hydrogels and their application in controlled release of polyphenols. Mater Sci Eng C 32:452-463. doi:10.1016/ j.msec.2011.11.018

Da Rosa CG, Borges CD, Zambiazi RC et al (2013) Microencapsulation of gallic acid in chitosan, beta-cyclodextrin and xanthan. Ind Crops Prod 46:138-146. doi:10.1016/j. indcrop.2012.12.053

Dandekar PP, Jain R, Patil S et al (2010) Curcumin-loaded hydrogel nanoparticles: application in anti-malarial therapy and toxicological evaluation. J Pharm Sci 99:49925010. doi:10.1002/jps.22191

Fang Z, Bhandari B (2010) Encapsulation of polyphenols-a review. Trends Food Sci Technol 21:510-523. doi:10. 1016/j.tifs.2010.08.003

Garcia-Fernandez MJ, Brackman G, Coenye T et al (2013) Antiseptic cyclodextrin-functionalized hydrogels and gauzes for loading and delivery of benzalkonium chloride. Biofouling 29:261-271. doi:10.1080/08927014.2013.765 947

Grice E, Segre J (2011) The skin microbiome. Nat Rev Microbiol 9:244-253. doi:10.1038/nrmicro2537

Guimaraes R, Barros L, Carvalho A, Ferreira ICFR (2010) Studies on chemical constituents and bioactivity of rosa micrantha: an alternative antioxidants source for food, pharmaceutical, or cosmetic applications. J Agric Food Chem 58:6277-6284. doi:10.1021/jf101394w

Gulrez SKH, Al-Assaf S, Phillips GO (2011) Hydrogels: methods of preparation, characterisation and applications. In: Carpi A (ed) Progress in molecular and environmental bioengineering-from analysis and modeling to technology applications, $1 \mathrm{st}$ edn. Inthech, Croatia, pp 117-150

Jones D, Lorimer C, McCoy C, Gorman S (2008) Characterization of the physicochemical, antimicrobial, and drug release properties of thermoresponsive hydrogel copolymers designed for medical device applications. J Biomed Mater Res B Appl Biomater 85:417-426. doi:10.1002/jbm. b. 30960

Lorenzo A, Rodríguez-Tenreiro C, Torres Labandeira JJ, Concheiro Nine A (2008) Method of obtaining hydrogels of cyclodextrins with glycidyl ethers, compositions thus obtained and applications Thereof. US Pat. App. 11/2
M2-A8 (2005) Padronização dos testes de sensibilidade a antimicrobianos por disco-difusão: Norma Aprovada. 23:1-58

Miranda T, Goff A Le, Pereira A, Soares G (2010) Studies on cotton modification with dodecenyl succinic anhydride (DDSA). In: Technology U of ZF of T (ed) 5th Int. Text. Cloth. Des. Conf.-Magic World Text. Dubrovnik, pp 1-6

Nicoletti A, Fiorini M, Paolillo J et al (2013) Effects of different crosslinking conditions on the chemical-physical properties of a novel bio-inspired composite scaffold stabilised with 1,4-butanediol diglycidyl ether (BDDGE). J Mater Sci Mater Med 24:17-35. doi:10.1007/s10856-012-4782-4

Pinho E (2014) Development of a new antimicrobial material for wound dressing. Universirty of Minho, Braga, Portugal

Pinho E, Grootveld M, Soares G, Henriques M (2013) Cyclodextrin-based hydrogels toward improved wound dressings. Crit Rev Biotechnol 8551:1-10. doi:10.3109/07388 551.2013.794413

Pinho E, Ferreira ICFR, Barros L et al (2014a) Antibacterial potential of northeastern portugal wild plant extracts and respective phenolic compounds. Biomed Res Int 2014: 814590. doi:10.1155/2014/814590

Pinho E, Grootveld M, Soares G, Henriques M (2014b) Cyclodextrins as encapsulation agents for plant bioactive compounds. Carbohydr Polym 101:121-135. doi:10.1016/ j.carbpol.2013.08.078

Rodriguez-Tenreiro C, Alvarez-Lorenzo C, Rodriguez-Perez A et al (2006) New cyclodextrin hydrogels cross-linked with diglycidylethers with a high drug loading and controlled release ability. Pharm Res 23:121-130. doi:10.1007/ s11095-005-8924-y

Rodriguez-Tenreiro C, Alvarez-Lorenzo C, Rodriguez-Perez A et al (2007) Estradiol sustained release from high affinity cyclodextrin hydrogels. Eur J Pharm Biopharm Off J Arbeitsgemeinschaft fur Pharm Verfahrenstechnik eV 66:55-62

Schwingel L, Fasolo D, Holzschuh M et al (2008) Association of 3-O-methylquercetin with $\beta$-cyclodextrin: complex preparation, characterization and ex vivo skin permeation studies. J Incl Phenom Macrocycl Chem 62:149-159. doi:10.1007/s10847-008-9450-4

Siepmann J, Peppas NA (2001) Modeling of drug release from delivery systems based on hydroxypropyl methylcellulose (HPMC). Adv Drug Deliv Rev 48:139-157

Sun R, Sun X, Tomkinson I (2003) Hemicelluloses and their derivatives. In: Gatenholm P, Tenkanen M (eds) Hemicelluloses: science and technology. American Chemical Society, Washington, DC, pp 2-22. doi:10.1021/bk-20040864.ch001

Thatiparti TR, Shoffstall AJ, Recum H (2010) Cyclodextrinbased device coatings for affinity-based release of antibiotics. Biomaterials 31:2335-2347. doi:10.1016/j.biomateri als.2009.11.087

Wang YX, Robertson JL, Spillman WB, Claus RO (2004) Effects of the chemical structure and the surface properties of polymeric biomaterials on their biocompatibility. Pharm Res 21:1362-1373

Wang X, Wang J, Yang N (2007) Flow injection chemiluminescent detection of gallic acid in olive fruits. Food Chem 105:340-345. doi:10.1016/j.foodchem.2006.11.061

Yasuda H, Sharma AK, Yasuda T (1981) Effect of orientation and mobility of polymer molecules at surfaces on contact 
angle and its hysteresis. J Polym Sci Polym Phys Ed 19:1285-1291. doi:10.1002/pol.1981.180190901

Yasuda T, Okuno T, Yasuda H (1994) Contact angle of water on polymer surfaces. Langmuir 10(7):2435-2439. doi:10. 1021/la00019a068

Zhang J-T, Huang S-W, Liu J, Zhuo R-X (2005) Temperature sensitive poly[N-isopropylacrylamide-co-(acryloyl betacyclodextrin)] for improved drug release. Macromol Biosci 5:192-196. doi:10.1002/mabi.200400167
Zhang L, Zhou J, Zhang L (2013) Structure and properties of $\beta$ cyclodextrin/cellulose hydrogels prepared in $\mathrm{NaOH} /$ urea aqueous solution. Carbohydr Polym 94:386-393. doi:10. 1016/j.carbpol.2012.12.077

Zugasti ME, Zornoza A, Goñi MDM et al (2009) Influence of soluble and insoluble cyclodextrin polymers on drug release from hydroxypropyl methylcellulose tablets. Drug Dev Ind Pharm 35:1264-1270. doi:10.1080/03639040902882306 